TAIWANESE JOURNAL OF MATHEMATICS

Vol. 11, No. 4, pp. 1127-1141, September 2007

This paper is available online at http://www.math.nthu.edu.tw/tjm/

\title{
VECTOR VALUED COMMUTATORS ON NON-HOMOGENEOUS SPACES
}

\author{
Wengu Chen and Changxing Miao
}

\begin{abstract}
Let $\mu$ be a Borel measure on $\mathbb{R}^{d}$ which may be non doubling. The only condition that $\mu$ must satisfy is $\mu(Q) \leq c_{0} l(Q)^{n}$ for any cube $Q \subset \mathbb{R}^{d}$ with sides parallel to the coordinate axes and for some fixed $n$ with $0<n \leq d$. This paper is to develop the vector valued commutator theory in the context of the non-homogeneous spaces. As an application, the boundedness of the maximal commutator of any Calderon-Zygmund operator on the non-homogeneous space with a $\operatorname{RBMO}(\mu)$ function introduced by Tolsa in [9] is obtained.
\end{abstract}

\section{INTRODUCTION}

Let $\mu$ be a non-negative $\mathrm{n}$-dimensional Borel measure on $R^{d}$, that is, a measure satisfying

$$
\mu(Q) \leq c_{0} l(Q)^{n}
$$

for any cube $Q \subset \mathbb{R}^{d}$ with sides parallel to the coordinate axes, where $l(Q)$ stands for the side length of $Q$ and $n$ is a fixed real number such that $0<n \leq d$. Throughout this paper, all cubes we shall consider will be those with sides parallel to the coordinate axes. For $r>0, r Q$ will denote the cube with the same center as $Q$ and with $l(r Q)=r l(Q)$. Moreover, $Q(x, r)$ will be the cube centered at $x$ with side length $r$.

The classical theory of harmonic analysis for maximal functions and singular integrals on $\left(\mathbb{R}^{n}, \mu\right)$ has been developed under the assumption that the underlying measure $\mu$ satisfies the doubling property, i.e., there exists a constant $c>0$ such that $\mu(B(x, 2 r)) \leq c \mu(B(x, r))$ for every $x \in \mathbb{R}^{n}$ and $r>0$. However, some recent

Accepted April 6, 2005.

Communicated by Yongsheng Han.

2000 Mathematics Subject Classification: 42B20, 42B25.

Key words and phrases: Calder on-Zygmund operator, Commutator non-doubling measure.

The work was partly supported by NNSF of China (No.10371080) and Special Funds for Major State

Basic Research Project of China. 
results on Calderon-Zygmund operators ([4,5,7,8]) and functions of bounded mean oscillation $([3,9])$ show that it should be possible to dispense with the doubling condition for most of the classical theory. The purpose of this paper is to develop the vector valued commutator theory in this new setting.

Let us introduce some notations and definitions. Given a Banach space $E$ we will denote by $L_{E}^{p}(\mu)$ the Bochner-Lebesgue space of $E$-valued strongly measurable functions such that

$$
\int_{\mathbb{R}^{d}}\|f(x)\|_{E}^{p} d \mu<+\infty .
$$

Given two cubes $Q \subset R$ in $\mathbb{R}^{d}$, we set

$$
K_{Q, R}=1+\sum_{k=1}^{N_{Q, R}} \frac{\mu\left(2^{k} Q\right)}{l\left(2^{k} Q\right)^{n}}
$$

where $N_{Q, R}$ is the first integer $k$ such that $l\left(2^{k} Q\right) \geq l(R) . K_{Q, R}$ was introduced by Tolsa in [9].

Given $\beta_{d}$ (depending on $d$ ) big enough (for example, $\beta_{d}>2^{n}$ ), we say that some cube $Q \subset \mathbb{R}^{d}$ is doubling if $\mu(2 Q) \leq \beta_{d} \mu(Q)$.

Given a cube $Q \subset \mathbb{R}^{d}$, let $N$ be the smallest integer $\geq 0$ such that $2^{N} Q$ is doubling. We denote this cube by $\tilde{Q}$.

Let $\eta>1$ be some fixed constant. We say that a locally integrable function $b(x)$ is in $R B M O(\mu)$ if there exists some constant $c_{1}$ such that for any cube $Q$,

$$
\frac{1}{\mu(\eta Q)} \int_{Q}\left|b-m_{\tilde{Q}} b\right| d \mu \leq c_{1}
$$

and

$$
\left|m_{Q} b-m_{R} b\right| \leq c_{1} K_{Q, R} \text { for any two doubling cubes } Q \subset R,
$$

where $m_{Q} b=\frac{1}{\mu(Q)} \int_{Q} b d \mu$. The minimal constant $c_{1}$ is the $R B M O(\mu)$ norm of $b$, and it will be denoted by $\|b\|_{*}$.

Let $E, F$ be a couple of Banach spaces. $\mathcal{L}(E, F)$ will denote the set of bounded linear operators from $E$ to $F$. We say a kernel $k(x, y): \mathbb{R}^{d} \times \mathbb{R}^{d} \backslash\{(x, y): x=$ $y\} \rightarrow \mathcal{L}(E, F)$ is a (vector-valued) $n$-dimensional singular integral kernel if

(1) $\|k(x, y)\|_{\mathcal{L}(E, F)} \leq \frac{A}{|x-y|^{n}}$ if $x \neq y$,

(2) and there exists $0<\delta \leq 1$ such that

$$
\left\|k(x, y)-k\left(x^{\prime}, y\right)\right\|_{\mathcal{L}(E, F)}+\left\|k(y, x)-k\left(y, x^{\prime}\right)\right\|_{\mathcal{L}(E, F)} \leq \frac{A\left|x-x^{\prime}\right|^{\delta}}{|x-y|^{n+\delta}}
$$


if $|x-y|>2\left|x-x^{\prime}\right|$.

A bounded linear operator $T$ from $L_{E}^{2}(\mu)$ to $L_{F}^{2}(\mu)$ is said to be a vector-valued Calderón-Zygmund operator with $n$-dimensional singular integral kernel $k$ if for every compactly supported function $f \in L_{E}^{2}(\mu)$,

$$
T f(x)=\int_{\mathbb{R}^{d}} k(x, y) f(y) d \mu(y), \text { for } x \notin \operatorname{supp} f .
$$

For $r>0$, we define the truncated operators by

$$
T_{r} f(x)=\int_{\mathbb{R}^{d} \backslash B(x, r)} k(x, y) f(y) d \mu(y)
$$

and define the maximal operator associated with $T$ as follows

$$
T_{*} f(x)=\sup _{r>0}\left\|T_{r} f(x)\right\|_{F} .
$$

\section{Cotlar Type Inequality and Boundedness of $T_{*}$}

Now we are ready to prove the boundedness of the maximal operator $T_{*}$. This follows immediately from

Theorem 1. Let $f \in L_{E}^{2}(\mu)$. For any $\beta>1$ and $x \in \operatorname{supp} \mu$,

$$
T_{*} f(x) \leq 4 \cdot 9^{n} \tilde{M}\left(\|T f\|_{F}\right)(x)+B(\beta) \tilde{M}_{\beta}\left(\|f\|_{E}\right)(x)
$$

where the constant $B(\beta)>0$ depends on the parameter $\beta>1$, the dimension $n$, the constants $\delta$ and $A$ in the definition of the singular integral kernel $k$, and the norm $\|T\|_{L_{E}^{2} \rightarrow L_{F}^{2}}$ only. $\tilde{M} f(x)=\sup _{r>0} \frac{1}{\mu(B(x, 3 r))} \int_{B(x, r)}|f(y)| d \mu(y)$ and $\tilde{M}_{\beta} f(x)=\left[\tilde{M}\left(|f|^{\beta}\right)(x)\right]^{1 / \beta}$.

Proof. We follow the ideas of [5, Theorem 7.1]. Let $x \in \operatorname{supp} \mu$ and $r>0$. Consider the sequence of balls $B\left(x, r_{j}\right)$ with $r_{j}=3^{j} r$ and set $\mu_{j}=\mu\left(B\left(x, r_{j}\right)\right)$. We can choose $l \geq 1$, the smallest positive integer such that $\mu_{l} \leq 2 \cdot 3^{n} \mu_{l-1}$. Put $R=r_{l-1}=3^{l-1} r$. Then

$$
\begin{aligned}
\left\|T_{r} f(x)-T_{3 R} f(x)\right\|_{F} \leq \int_{B(x, 3 R) \backslash B(x, r)}\|k(x, y)\|_{\mathcal{L}(E, F)}\|f(y)\|_{E} d \mu(y) \\
\quad=\sum_{j=1}^{l} \int_{B\left(x, r_{j}\right) \backslash B\left(x, r_{j-1}\right)}\|k(x, y)\|_{\mathcal{L}(E, F)}\|f(y)\|_{E} d \mu(y) \\
\quad \leq \sum_{j=1}^{l} A r_{j-1}^{-n} \int_{B\left(x, r_{j}\right)}\|f(y)\|_{E} d \mu(y) .
\end{aligned}
$$


Note that $r_{j-1}=3^{j-1-l} r_{l}$ and

$$
\mu_{j} \leq\left(2 \cdot 3^{n}\right)^{j-l+1} \mu_{l} \quad \text { for } \quad 1 \leq j \leq l .
$$

Hence

$$
\begin{aligned}
& \left\|T_{r} f(x)-T_{3 R} f(x)\right\|_{F} \leq \sum_{j=1}^{l} A r_{j-1}^{-n} \mu_{j} \tilde{M}\left(\|f\|_{E}\right)(x) \\
& \quad \leq 2 \cdot 9^{n} A \tilde{M}\left(\|f\|_{E}\right)(x) \frac{\mu_{l}}{r_{l}^{n}} \sum_{j=1}^{l} 2^{j-l} \leq 4 \cdot 9^{n} A \tilde{M}\left(\|f\|_{E}\right)(x) .
\end{aligned}
$$

So, we need only to estimate $T_{3 R} f(x)$. We consider the average

$$
V_{R}(x):=\frac{1}{\mu(B(x, R))} \int_{B(x, R)} T f d \mu,
$$

which is bounded by $\frac{\mu(B(x, 3 R))}{\mu(B(x, R))} \tilde{M}\left(\|T f\|_{F}\right)(x) \leq 4 \cdot 9^{n} \tilde{M}\left(\|T f\|_{F}\right)(x)$. On the other hand,

$$
\begin{aligned}
V_{R}(x) & =\frac{1}{\mu(B(x, R))} \int_{B(x, R)} T\left(f \chi_{\mathbb{R}^{d} \backslash B(x, 3 R)}\right) d \mu \\
& +\frac{1}{\mu(B(x, R))} \int_{B(x, R)} T\left(f \chi_{B(x, 3 R)}\right) d \mu=I+I I,
\end{aligned}
$$

and $\left\|T_{3 R} f(x)-V_{R}(x)\right\|_{F} \leq\left\|T_{3 R} f(x)-I\right\|_{F}+\|I I\|_{F}$. By using the second condition on the kernel,

$$
\begin{aligned}
& \left\|T_{3 R} f(x)-I\right\|_{F} \\
& \leq \frac{1}{\mu(B(x, R))} \int_{B(x, R)} \int_{\mathbb{R}^{d} \backslash B(x, 3 R)}\|k(x, y)-k(z, y)\|_{\mathcal{L}(E, F)}\|f(y)\|_{E} d \mu(y) d \mu(z) \\
& \leq \frac{1}{\mu(B(x, R))} \int_{B(x, R)} \sum_{j=1}^{\infty} \int_{B\left(x, 3^{j+1} R\right) \backslash B(x, 3 j R)} \frac{A|x-z|^{\delta}}{|y-x|^{n+\delta}}\|f(y)\|_{E} d \mu(y) d \mu(z) \\
& \leq \frac{1}{\mu(B(x, R))} \int_{B(x, R)} \sum_{j=1}^{\infty} \frac{A R^{\delta}}{\left(3^{j} R\right)^{n+\delta}} \int_{B\left(x, 3^{j+1} R\right)}\|f(y)\|_{E} d \mu(y) d \mu(z) \\
& \quad \leq c(\delta) 3^{n} A \tilde{M}\left(\|f\|_{E}\right)(x) .
\end{aligned}
$$

Whereas for the second term, by Hölder's inequality,

$$
\begin{aligned}
\|I I\|_{F} & \leq \frac{1}{\mu(B(x, R))}\|T\|_{L_{E}^{\beta}(\mu) \rightarrow L_{F}^{\beta}(\mu)} \mu(B(x, R))^{1 / \beta^{\prime}}\left\|f \chi_{B(x, 3 R)}\right\|_{L_{E}^{\beta}(\mu)} \\
& \leq\|T\|_{L_{E}^{\beta}(\mu) \rightarrow L_{F}^{\beta}(\mu)}\left(\frac{1}{\mu(B(x, R))} \int_{B(x, 3 R)}\|f(y)\|_{E}^{\beta} d \mu\right)^{1 / \beta} .
\end{aligned}
$$


According to our choice of $R$, we have

$$
\mu(B(x, 3 R))=\mu_{l} \leq 2 \cdot 3^{n} \mu_{l-1}=2 \cdot 3^{n} \mu(B(x, R)) .
$$

This allows us to conclude that the second term is bounded by

$$
\left(2 \cdot 3^{n}\right)^{1 / \beta}\|T\|_{L_{E}^{\beta}(\mu) \rightarrow L_{F}^{\beta}(\mu)} \tilde{M}_{\beta}\left(\|f\|_{E}\right)(x) .
$$

By taking the supremum on $r>0$ we have the desired estimate.

\section{The Vector Valued Commutator Theorems}

Now we can state the main results in this paper.

Theorem 2. Let E, F be Banach spaces. Let $T$ be a vector-valued CalderonZygmund operator with an $n$-dimensional singular integral kernel $k(x, y)$. Given $b \in R B M O(\mu)$. Then the commutator $C_{b}$ defined by

$$
C_{b} f(x)=b(x) T f(x)-T(b f)(x)
$$

is bounded from $L_{E}^{p}(\mu)$ into $L_{F}^{p}(\mu)$ for $1<p<\infty$.

Theorem 2. Let $F$ be a Banach lattice and $V$ a bounded linear operator from $L^{p}(\mu)$ into $L_{F}^{p}(\mu)$ for $1<p<\infty$. Assume that there exists an $F$-valued function $w(x, y)$ satisfying:

(W1) for any compactly supported function $f \in L^{2}(\mu)$,

$$
V f(x)=\int_{\mathbb{R}^{d}} w(x, y) f(y) d \mu(y),
$$

$(W 2)$ for every $x$ and $y \in \mathbb{R}^{d}, w(x, y) \geq 0$, and for $x \neq y$,

$$
\|w(x, y)\|_{F} \leq \frac{A}{|x-y|^{n}}
$$

(W3) there exists $0<\delta \leq 1$ such that

$$
\begin{gathered}
\left\|w(x, y)-w\left(x^{\prime}, y\right)\right\|_{F}+\left\|w(y, x)-w\left(y, x^{\prime}\right)\right\|_{F} \leq \frac{A\left|x-x^{\prime}\right|^{\delta}}{|x-y|^{n+\delta}} \\
\text { if }|x-y|>2\left|x-x^{\prime}\right| . \text { If } b \in R B M O(\mu), \text { then the operator } V_{b}^{+} \text {defined by } \\
V_{b}^{+} f(x)=\int_{\mathbb{R}^{d}}|b(x)-b(y)| w(x, y) f(y) d \mu(y)
\end{gathered}
$$

is bounded from $L^{p}(\mu)$ into $L_{F}^{p}(\mu)$ for $1<p<\infty$. 


\section{An Application of the Vector Valued Commutator Theorems}

Let $b \in R B M O(\mu)$ and $T$ be a Calderon-Zygmund operator with an $n$-dimensional singular integral kernel $k(x, y)$. We define

$$
T_{\epsilon} f(x)=\int_{|x-y|>\epsilon} k(x, y) f(y) d \mu(y),
$$

and

$$
C_{b}^{*} f(x)=\sup _{\epsilon>0}\left|b(x) T_{\epsilon} f(x)-T_{\epsilon}(b f)(x)\right| .
$$

Then the operator $C_{b}^{*}$ is bounded on $L^{p}(\mu)$ for all $1<p<\infty$.

Following the idea of [6], we take $\phi, \psi \in C^{\infty}([0, \infty))$ such that $\left|\phi^{\prime}(t)\right| \leq$ $c t^{-1},\left|\psi^{\prime}(t)\right| \leq c t^{-1}$ and

$$
\chi_{[2, \infty)} \leq \phi \leq \chi_{[1, \infty)}, \chi_{[1,2]} \leq \psi \leq \chi_{[1 / 2,3]} .
$$

We consider the operators

$$
\Phi f(x)=\left\{\phi_{\epsilon} f(x)\right\}_{\epsilon>0}=\left\{\int k(x, y) \phi\left(\frac{|x-y|}{\epsilon}\right) f(y) d \mu(y)\right\}_{\epsilon>0}
$$

and

$$
\Psi f(x)=\left\{\psi_{\epsilon} f(x)\right\}_{\epsilon>0}=\left\{\int|k(x, y)| \psi\left(\frac{|x-y|}{\epsilon}\right) f(y) d \mu(y)\right\}_{\epsilon>0},
$$

with kernels given by

$$
\left\{\phi_{\epsilon}(x, y)\right\}_{\epsilon>0}=\left\{k(x, y) \phi\left(\frac{|x-y|}{\epsilon}\right)\right\}_{\epsilon>0}
$$

and

$$
\left\{\psi_{\epsilon}(x, y)\right\}_{\epsilon>0}=\left\{|k(x, y)| \psi\left(\frac{|x-y|}{\epsilon}\right)\right\}_{\epsilon>0} .
$$

The kernel of $\Phi$ as $l^{\infty}(\mathbb{R})$-valued function is an $n$-dimensional singular integral kernel. Analogously, it can be shown that the kernel of $\Psi$ satisfies (W2) and (W3) of Theorem 3. By the vector valued Calderon-Zygmund theory on the nonhomogeneous spaces, see [1, 2], $\Phi$ and $\Psi$ are bounded linear operators from $L^{p}(\mu)$ into $L_{l^{\infty}}^{p}(\mu)$ for all $1<p<\infty$. Therefore $\Phi$ satisfies the hypotheses of Theorem 2 and $\Psi$ satisfies the hypotheses of Theorem 3. Then by Theorems 2 and 3 the operators

$$
\Phi_{b} f(x)=\left\{b(x) \phi_{\epsilon} f(x)-\phi_{\epsilon}(b f)(x)\right\}_{\epsilon>0}
$$


and

$$
\Psi_{b}^{+} f(x)=\left\{\int|b(x)-b(y)| \psi_{\epsilon}(x, y) f(y) d \mu(y)\right\}_{\epsilon>0}
$$

are bounded from $L^{p}(\mu)$ into $L_{l^{\infty}}^{p}(\mu)$ for all $1<p<\infty$.

Now, we consider the operator

$$
\tilde{T}_{b} f(x)=\left\{b(x) T_{\epsilon} f(x)-T_{\epsilon}(b f)(x)\right\}_{\epsilon>0} .
$$

The difference operator

$$
\begin{aligned}
& U_{b} f(x)=\Phi_{b} f(x)-\tilde{T}_{b} f(x) \\
& \quad=\left\{\int(b(x)-b(y))\left[\phi\left(\frac{|x-y|}{\epsilon}\right)-\chi_{[1, \infty)}\left(\frac{|x-y|}{\epsilon}\right)\right] k(x, y) f(y) d \mu(y)\right\}_{\epsilon>0}
\end{aligned}
$$

satisfies, for a certain $\psi$ as above, that

$$
\begin{aligned}
& \left\|U_{b} f(x)\right\|_{l^{\infty}} \leq \sup _{\epsilon>0} \int|b(x)-b(y)||k(x, y)| \psi\left(\frac{|x-y|}{\epsilon}\right)|f(y)| d \mu(y) \\
& =\left\|\Psi_{b}^{+} f(x)\right\|_{l^{\infty}}
\end{aligned}
$$

and therefore $U_{b}$ is bounded from $L^{p}(\mu)$ into $L_{l \infty}^{p}(\mu)$ and, consequently, $\tilde{T}_{b}$ is bounded from $L^{p}(\mu)$ into $L_{l^{\infty}}^{p}(\mu)$, that is to say $C_{b}^{*}$ is bounded on $L^{p}(\mu)$.

\section{The Proof of the Vector Valued Commutator Theorems}

Before proving the theorems, we need another equivalent norm for $R B M O(\mu)$ and some lemmas.

Suppose that for a measurable function $b(x)$ there exists some $c_{2}$ and for each cube $Q$, there exists real number $b_{Q}$ such that

$$
\sup _{Q} \frac{1}{\mu(\eta Q)} \int_{Q}\left|b-b_{Q}\right| d \mu \leq c_{2}
$$

and

$$
\left|b_{Q}-b_{R}\right| \leq c_{2} K_{Q, R} \text { for any two cubes } Q \subset R .
$$

Then, we write $\|b\|_{* *}=\inf c_{2}$, where the infimum is taken over all the constants $c_{2}$ and all the numbers $\left\{b_{Q}\right\}$ satisfying (3) and (4). By Lemma 2.8 in [9], the norms $\|\cdot\|_{*}$ and $\|\cdot\|_{* *}$ are equivalent for a fixed $\eta>1$.

In [9], Tolsa defined a sharp maximal operator $M^{\#} f(x)$ such that

$$
f \in R B M O(\mu) \Longleftrightarrow M^{\#} f \in L^{\infty}(\mu) .
$$


In order to prove the theorems, we need to introduce the vector valued version $M_{E}^{\#} f(x)$. We define

$$
M_{E}^{\#} f(x)=\sup _{x \in Q} \frac{1}{\mu\left(\frac{3}{2} Q\right)} \int_{Q}\left\|f-m_{\tilde{Q}} f\right\|_{E} d \mu+\sup _{\substack{x \in Q \subset R \\ Q, R \text { doubling }}} \frac{\left\|m_{Q} f-m_{R} f\right\|_{E}}{K_{Q, R}} .
$$

We also consider the non-centered doubling maximal operator $N_{E}$ :

$$
N_{E} f(x)=\sup _{\substack{x \in Q \\ Q \text { doubling }}} \frac{1}{\mu(Q)} \int_{Q}\|f\|_{E} d \mu .
$$

By the Remark 2.3 of [9], for $\mu$-almost all $x \in \mathbb{R}^{d}$ one can find a sequence of doubling cubes $\left\{Q_{k}\right\}_{k}$ centered at $x$ with $l\left(Q_{k}\right) \rightarrow 0$ as $k \rightarrow \infty$ such that

$$
\lim _{k \rightarrow \infty} \frac{1}{\mu\left(Q_{k}\right)} \int_{Q_{k}} b(y) d \mu(y)=b(x) .
$$

So, $\|f(x)\|_{E} \leq N_{E} f(x)$ for $\mu$ - a.e. $x \in \mathbb{R}^{d}$. Moreover, it is easy to show that $N_{E}$ is bounded from $L_{E}^{1}(\mu)$ into $L^{1, \infty}(\mu)$ and from $L_{E}^{p}(\mu)$ into $L^{p}(\mu)$ for $p \in(1, \infty]$.

Lemma 1. Let $f(x)$ be an E-valued strongly measurable function with $\int f d \mu=0$ if $\|\mu\|<\infty$. For $1<p<\infty$, if $\inf \left(1, N_{E} f\right) \in L^{p}(\mu)$, then we have

$$
\left\|N_{E} f\right\|_{L^{p}(\mu)} \leq c\left\|M_{E}^{\#} f\right\|_{L^{p}(\mu)} .
$$

For the scalar case, this is the theorem 6.2 of [9]. By some modifications one can obtain the proof of the lemma. We omit the proof here for brevity.

Proof of the theorem 2. For all $p \in(1, \infty)$, we will show the following sharp maximal function estimate

$M_{F}^{\#}\left(C_{b} f\right)(x) \leq c_{p}\|b\|_{*}\left(M_{p,(9 / 8)}\left(\|f\|_{E}\right)(x)+M_{p,(3 / 2)}\left(\|T f\|_{F}\right)(x)+T_{*} f(x)\right)$,

where, for $\eta>1, M_{p,(\eta)}$ is the non-centered maximal operator

$$
M_{p,(\eta)} f(x)=\sup _{x \in Q}\left(\frac{1}{\mu(\eta Q)} \int_{Q}|f|^{p} d \mu\right)^{1 / p}
$$

and the operator $M_{p,(\eta)}$ is bounded on $L^{r}(\mu)$ for $r>p$. 
By Theorem 1 and Lemma 1 , if we take $r$ such that $1<r<p<\infty$, we can get

$$
\begin{aligned}
& \left\|C_{b} f\right\|_{L_{F}^{p}(\mu)} \leq\left\|N_{F}\left(C_{b} f\right)\right\|_{L^{p}(\mu)} \leq c\left\|M_{F}^{\#}\left(C_{b} f\right)\right\|_{L^{p}(\mu)} \\
& \quad \leq c\|b\|_{*}\left(\left\|M_{r,(9 / 8)}\left(\|f\|_{E}\right)\right\|_{L^{p}(\mu)}+\left\|M_{r,(3 / 2)}\left(\|T f\|_{F}\right)\right\|_{L^{p}(\mu)}+\left\|T_{*} f\right\|_{L^{p}(\mu)}\right) \\
& \quad \leq c\|b\|_{*}\|f\|_{L_{E}^{p}(\mu)} .
\end{aligned}
$$

Now we remain to show the above sharp maximal function estimate.

Let $\left\{b_{Q}\right\}_{Q}$ be a family of numbers satisfying

$$
\int_{Q}\left|b-b_{Q}\right| d \mu \leq 2 \mu(2 Q)\|b\|_{* *}
$$

for any cube $Q$, and

$$
\left|b_{Q}-b_{R}\right| \leq 2 K_{Q, R}\|b\|_{* *}
$$

for all cubes $Q \subset R$. For any cube $Q$, we denote

$$
h_{Q}:=m_{Q}\left(T\left(\left(b-b_{Q}\right) f \chi_{\mathbb{R}^{d} \backslash \frac{4}{3} Q}\right)\right) .
$$

We will prove that

$$
\begin{gathered}
\frac{1}{\mu\left(\frac{3}{2} Q\right)} \int_{Q}\left\|C_{b} f-h_{Q}\right\|_{F} d \mu \leq c\|b\|_{*}\left(M_{p,(9 / 8)}\left(\|f\|_{E}\right)(x)\right. \\
\left.+M_{p,(3 / 2)}\left(\|T f\|_{F}\right)(x)\right)
\end{gathered}
$$

for all $x$ and $Q$ with $x \in Q$, and

$$
\left\|h_{Q}-h_{R}\right\|_{F} \leq c\|b\|_{*}\left(M_{p,(9 / 8)}\left(\|f\|_{E}\right)(x)+T_{*} f(x)\right) K_{Q, R}^{2}
$$

for all cubes $Q \subset R$ with $x \in Q$.

To get (5) for some fixed cube $Q$ and $x$ with $x \in Q$, we write $C_{b} f$ in the following way:

$$
C_{b} f=\left(b-b_{Q}\right) T f-T\left(\left(b-b_{Q}\right) f_{1}\right)-T\left(\left(b-b_{Q}\right) f_{2}\right),
$$

where $f_{1}=f \chi_{\frac{4}{3} Q}$ and $f_{2}=f-f_{1}$. Let us estimate the term $\left(b-b_{Q}\right) T f$ :

$$
\begin{aligned}
& \frac{1}{\mu\left(\frac{3}{2} Q\right)} \int_{Q}\left\|\left(b-b_{Q}\right) T f\right\|_{F} d \mu \leq\left(\frac{1}{\mu\left(\frac{3}{2} Q\right)} \int_{Q}\left|b-b_{Q}\right|^{p^{\prime}} d \mu\right)^{1 / p^{\prime}} \\
& \quad \times\left(\frac{1}{\mu\left(\frac{3}{2} Q\right)} \int_{Q}\|T f\|_{F}^{p} d \mu\right)^{1 / p} \\
& \quad \leq\left(\frac{1}{\mu\left(\frac{3}{2} Q\right)} \int_{Q}\left|b-b_{Q}\right|^{p^{\prime}} d \mu\right)^{1 / p^{\prime}} M_{p,(3 / 2)}\left(\|T f\|_{F}\right)(x) \\
& \quad \leq c\|b\|_{*} M_{p,(3 / 2)}\left(\|T f\|_{F}\right)(x) .
\end{aligned}
$$


Now we are going to estimate the second term on the right hand side of (7). We take $s=\sqrt{p}$. Then we have

$$
\begin{aligned}
& \frac{1}{\mu\left(\frac{3}{2} Q\right)} \int_{Q}\left\|T\left(\left(b-b_{Q}\right) f_{1}\right)\right\|_{F} d \mu \leq \frac{\mu(Q)^{1-1 / s}}{\mu\left(\frac{3}{2} Q\right)}\left\|T\left(\left(b-b_{Q}\right) f_{1}\right)\right\|_{L_{F}^{s}(\mu)} \\
& \quad \leq c \frac{\mu(Q)^{1-1 / s}}{\mu\left(\frac{3}{2} Q\right)}\left\|\left(b-b_{Q}\right) f_{1}\right\|_{L_{E}^{s}(\mu)} \\
& \quad \leq c \frac{\mu(Q)^{1-1 / s}}{\mu\left(\frac{3}{2} Q\right)}\left(\int_{\frac{4}{3} Q}\left\|\left(b-b_{Q}\right) f_{1}\right\|_{E}^{s} d \mu\right)^{1 / s} \\
& \leq c \frac{1}{\mu\left(\frac{3}{2} Q\right)^{1 / s}}\left(\int_{\frac{4}{3} Q}\left|b-b_{Q}\right|^{s s^{\prime}} d \mu\right)^{1 / s s^{\prime}}\left(\int_{\frac{4}{3} Q}\|f\|_{E}^{p} d \mu\right)^{1 / p} \\
& \leq c\|b\|_{*} M_{p,(9 / 8)}\left(\|f\|_{E}\right)(x) .
\end{aligned}
$$

(9)

By (7), (8) and (9), to get (5) we only need to estimate the difference $\| T((b-$ $\left.\left.b_{Q}\right) f_{2}\right)-h_{Q} \|_{F}$. For $y_{1}, y_{2} \in Q$ we have

$$
\begin{aligned}
\| & T\left(\left(b-b_{Q}\right) f_{2}\right)\left(y_{1}\right)-T\left(\left(b-b_{Q}\right) f_{2}\right)\left(y_{2}\right) \|_{F} \\
\leq & c \int_{\mathbb{R}^{d} \backslash \frac{4}{3} Q} \frac{\left|y_{2}-y_{1}\right|^{\delta}}{\left|z-y_{1}\right|^{n+\delta}}\left|b(z)-b_{Q}\right|\|f(z)\|_{E} d \mu(z) \\
\leq & c \sum_{k=1}^{\infty} \int_{2^{k} \frac{4}{3} Q \backslash 2^{k-1} \frac{4}{3} Q} \frac{l(Q)^{\delta}}{\left|z-y_{1}\right|^{n+\delta}}\left(\left|b(z)-b_{2^{k} \frac{4}{3} Q}\right|\right. \\
& \left.+\left|b_{Q}-b_{2^{k} \frac{4}{3} Q}\right|\right)\|f(z)\|_{E} d \mu(z) \\
\leq & c \sum_{k=1}^{\infty} 2^{-k \delta} \frac{1}{l\left(2^{k} Q\right)^{n}} \int_{2^{k} \frac{4}{3} Q}\left|b(z)-b_{2^{k} \frac{4}{3} Q}\right|\|f(z)\|_{E} d \mu(z) \\
& +c \sum_{k=1}^{\infty} k 2^{-k \delta}\|b\|_{*} \frac{1}{l\left(2^{k} Q\right)^{n}} \int_{2^{k} \frac{4}{3} Q}\|f(z)\|_{E} d \mu(z) \\
\leq & c \sum_{k=1}^{\infty} 2^{-k \delta}\left(\frac{1}{\mu\left(2^{k} \frac{3}{2} Q\right)} \int_{2^{k} \frac{4}{3} Q}\left|b-b_{2^{k} \frac{4}{3} Q}\right|^{p^{\prime}} d \mu\right)^{1 / p^{\prime}} \\
& \times\left(\frac{1}{\mu\left(2^{k} \frac{3}{2} Q\right)} \int_{2^{k} \frac{4}{3} Q}\|f\|_{E}^{p} d \mu\right)^{1 / p} \\
& +c \sum_{k=1}^{\infty} k 2^{-k \delta}\|b\|_{*}\left(\frac{1}{\mu\left(2^{k} \frac{3}{2} Q\right)} \int_{2^{k} \frac{4}{3} Q}\|f\|_{E}^{p} d \mu\right)^{1 / p}
\end{aligned}
$$




$$
\begin{aligned}
& \leq c \sum_{k=1}^{\infty} 2^{-k \delta}\|b\|_{*} M_{p,(9 / 8)}\left(\|f\|_{E}\right)(x) \\
& \quad+c \sum_{k=1}^{\infty} k 2^{-k \delta}\|b\|_{*} M_{p,(9 / 8)}\left(\|f\|_{E}\right)(x) \\
& \leq c\|b\|_{*} M_{p,(9 / 8)}\left(\|f\|_{E}\right)(x),
\end{aligned}
$$

where we used the fact that

$$
\left|b_{Q}-b_{2^{k} \frac{4}{3} Q}\right| \leq 2 K_{Q, 2^{k} \frac{4}{3} Q}\|b\|_{* *} \leq c k\|b\|_{*} .
$$

Taking the mean over $y_{2} \in Q$, we get

$$
\begin{aligned}
& \left\|T\left(\left(b-b_{Q}\right) f_{2}\right)\left(y_{1}\right)-h_{Q}\right\|_{F}=\left\|T\left(\left(b-b_{Q}\right) f_{2}\right)\left(y_{1}\right)-m_{Q}\left(T\left(\left(b-b_{Q}\right) f_{2}\right)\right)\right\|_{F} \\
& \quad \leq c\|b\|_{*} M_{p,(9 / 8)}\left(\|f\|_{E}\right)(x) .
\end{aligned}
$$

Then

$$
\begin{aligned}
& \frac{1}{\mu\left(\frac{3}{2} Q\right)} \int_{Q}\left\|T\left(\left(b-b_{Q}\right) f_{2}\right)\left(y_{1}\right)-h_{Q}\right\|_{F} d \mu\left(y_{1}\right) \\
& \quad \leq c\|b\|_{*} M_{p,(9 / 8)}\left(\|f\|_{E}\right)(x)
\end{aligned}
$$

and so (5) holds.

Now we have to check the regularity condition (6) for the elements $\left\{h_{Q}\right\}_{Q}$. Consider two cubes $Q \subset R$ with $x \in Q$. We denote $N=N_{Q, R}+1$. We write the difference $\left\|h_{Q}-h_{R}\right\|_{F}$ in the following way:

$$
\begin{aligned}
\| m_{Q} & \left(T\left(\left(b-b_{Q}\right) f \chi_{\mathbb{R}^{d} \backslash \frac{4}{3} Q}\right)\right)-m_{R}\left(T\left(\left(b-b_{R}\right) f \chi_{\mathbb{R}^{d} \backslash \frac{4}{3} R}\right)\right) \|_{F} \\
\leq & \left\|m_{Q}\left(T\left(\left(b-b_{Q}\right) f \chi_{2 Q \backslash \frac{4}{3} Q}\right)\right)\right\|_{F}+\left\|m_{Q}\left(T\left(\left(b_{Q}-b_{R}\right) f \chi_{\mathbb{R}^{d} \backslash 2 Q}\right)\right)\right\|_{F} \\
& +\left\|m_{Q}\left(T\left(\left(b-b_{R}\right) f \chi_{2^{N} Q \backslash 2 Q}\right)\right)\right\|_{F}+\left\|m_{R}\left(T\left(\left(b-b_{R}\right) f \chi_{2^{N} Q \backslash \frac{4}{3} R}\right)\right)\right\|_{F} \\
& +\left\|m_{Q}\left(T\left(\left(b-b_{R}\right) f \chi_{\mathbb{R}^{d} \backslash 2^{N} Q}\right)\right)-m_{R}\left(T\left(\left(b-b_{R}\right) f \chi_{\mathbb{R}^{d} \backslash 2^{N} Q}\right)\right)\right\|_{F} \\
:= & U_{1}+U_{2}+U_{3}+U_{4}+U_{5} .
\end{aligned}
$$

Let us estimate $U_{1}$. For $y \in Q$ we have

$$
\begin{gathered}
\left\|T\left(\left(b-b_{Q}\right) f \chi_{2 Q \backslash \frac{4}{3} Q}\right)(y)\right\|_{F} \leq \frac{c}{l(Q)^{n}} \int_{2 Q}\left|b-b_{Q}\right|\|f\|_{E} d \mu \\
\leq \frac{c}{l(Q)^{n}}\left(\int_{2 Q}\left|b-b_{Q}\right|^{p^{\prime}} d \mu\right)^{1 / p^{\prime}}\left(\int_{2 Q}\|f\|_{E}^{p} d \mu\right)^{1 / p}
\end{gathered}
$$




$$
\begin{aligned}
& \leq c\left(\frac{1}{\mu(3 Q)} \int_{2 Q}\left|b-b_{Q}\right|^{p^{\prime}} d \mu\right)^{1 / p^{\prime}}\left(\frac{1}{\mu\left(\frac{9}{4} Q\right)} \int_{2 Q}\|f\|_{E}^{p} d \mu\right)^{1 / p} \\
& \leq c\|b\|_{*} M_{p,(9 / 8)}\left(\|f\|_{E}\right)(x) .
\end{aligned}
$$

So we obtain $U_{1} \leq c\|b\|_{*} M_{p,(9 / 8)}\left(\|f\|_{E}\right)(x)$.

Let us consider the term $U_{2}$. For $x, y \in Q$, it is easily seen that

$$
\left\|T\left(f \chi_{\mathbb{R}^{d} \backslash 2 Q}\right)(y)\right\|_{F} \leq T_{*} f(x)+c M_{p,(9 / 8)}\left(\|f\|_{E}\right)(x) .
$$

Thus

$$
\begin{aligned}
U_{2} & =\left\|\frac{1}{\mu(Q)} \int_{Q}\left(b_{Q}-b_{R}\right) T\left(f \chi_{\mathbb{R}^{d} \backslash 2 Q}\right)(y) d \mu\right\|_{F} \\
& \leq c K_{Q, R}\|b\|_{*}\left(T_{*} f(x)+M_{p,(9 / 8)}\left(\|f\|_{E}\right)(x)\right) .
\end{aligned}
$$

The term $U_{4}$ is easy to estimate. Some calculations similar to the ones for $U_{1}$ yield $U_{4} \leq c\|b\|_{*} M_{p,(9 / 8)}\left(\|f\|_{E}\right)(x)$.

Let us turn to estimate the term $U_{5}$. Operating as in (10), for any $y, z \in R$, we get

$\left\|T\left(\left(b-b_{R}\right) f \chi_{\mathbb{R}^{d} \backslash 2^{N} Q}\right)(y)-T\left(\left(b-b_{R}\right) f \chi_{\mathbb{R}^{d} \backslash 2^{N} Q}\right)(z)\right\|_{F} \leq c\|b\|_{*} M_{p,(9 / 8)}\left(\|f\|_{E}\right)(x)$.

Taking the mean over $Q$ for $y$ and over $R$ for $z$, we obtain

$$
U_{5} \leq c\|b\|_{*} M_{p,(9 / 8)}\left(\|f\|_{E}\right)(x) .
$$

Finally, we remain to deal with $U_{3}$. For $y \in Q$, we have

$$
\begin{aligned}
& \left\|T\left(\left(b-b_{R}\right) f \chi_{2^{N} Q \backslash 2 Q}\right)(y)\right\|_{F} \leq c \sum_{k=1}^{N-1} \frac{1}{l\left(2^{k} Q\right)^{n}} \int_{2^{k+1} Q \backslash 2^{k} Q}\left|b-b_{R}\right|\|f\|_{E} d \mu \\
& \leq c \sum_{k=1}^{N-1} \frac{1}{l\left(2^{k} Q\right)^{n}}\left(\int_{2^{k+1} Q}\left|b-b_{R}\right|^{p^{\prime}} d \mu\right)^{1 / p^{\prime}}\left(\int_{2^{k+1} Q}\|f\|_{E}^{p} d \mu\right)^{1 / p} .
\end{aligned}
$$

Note that

$$
\begin{aligned}
& \left(\int_{2^{k+1} Q}\left|b-b_{R}\right|^{p^{\prime}} d \mu\right)^{1 / p^{\prime}} \\
& \leq\left(\int_{2^{k+1} Q}\left|b-b_{2^{k+1} Q}\right|^{p^{\prime}} d \mu\right)^{1 / p^{\prime}}+\mu\left(2^{k+1} Q\right)^{1 / p^{\prime}}\left|b_{2^{k+1} Q}-b_{R}\right| \\
& \leq c K_{Q, R}\|b\|_{*} \mu\left(2^{k+2} Q\right)^{1 / p^{\prime}} .
\end{aligned}
$$


Thus

$$
\begin{aligned}
& \left\|T\left(\left(b-b_{R}\right) f \chi_{2^{N} Q \backslash 2 Q}\right)(y)\right\|_{F} \\
& \quad \leq c K_{Q, R}\|b\|_{*} \sum_{k=1}^{N-1} \frac{\mu\left(2^{k+2} Q\right)^{1 / p^{\prime}}}{l\left(2^{k} Q\right)^{n}}\left(\int_{2^{k+1} Q}\|f\|_{E}^{p} d \mu\right)^{1 / p} \\
& \quad \leq c K_{Q, R}\|b\|_{*} \sum_{k=1}^{N_{Q, R}} \frac{\mu\left(2^{k+2} Q\right)}{l\left(2^{k} Q\right)^{n}}\left(\frac{1}{\mu\left(2^{k+2} Q\right)} \int_{2^{k+1} Q}\|f\|_{E}^{p} d \mu\right)^{1 / p} \\
& \quad \leq c K_{Q, R}^{2}\|b\|_{*} M_{p,(9 / 8)}\left(\|f\|_{E}\right)(x) .
\end{aligned}
$$

Taking the mean over $Q$, we get

$$
U_{3} \leq c K_{Q, R}^{2}\|b\|_{*} M_{p,(9 / 8)}\left(\|f\|_{E}\right)(x) .
$$

So by the estimates on $U_{1}, U_{2}, U_{3}, U_{4}$ and $U_{5}$, the regularity condition (6) follows.

Let us see how from (5) and (6) one obtain the sharp maximal function estimate.

From (5), if $Q$ is a doubling cube and $x \in Q$, we have

$$
\begin{aligned}
& \left\|m_{Q}\left(C_{b} f\right)-h_{Q}\right\|_{F} \leq \frac{1}{\mu(Q)} \int_{Q}\left\|C_{b} f-h_{Q}\right\|_{F} d \mu \\
& \quad \leq c\|b\|_{*}\left(M_{p,(9 / 8)}\left(\|f\|_{E}\right)(x)+M_{p,(3 / 2)}\left(\|T f\|_{F}\right)(x)\right) .
\end{aligned}
$$

Also, for any cube $Q \ni x, K_{Q, \tilde{Q}} \leq c$, and then by (5) and (6) we get

$$
\begin{aligned}
& \frac{1}{\mu\left(\frac{3}{2} Q\right)} \int_{Q}\left\|C_{b} f-m_{\tilde{Q}}\left(C_{b} f\right)\right\|_{F} d \mu \\
& \quad \leq \frac{1}{\mu\left(\frac{3}{2} Q\right)} \int_{Q}\left\|C_{b} f-h_{Q}\right\|_{F} d \mu+\left\|h_{Q}-h_{\tilde{Q}}\right\|_{F}+\left\|h_{\tilde{Q}}-m_{\tilde{Q}}\left(C_{b} f\right)\right\|_{F} \\
& \quad \leq c\|b\|_{*}\left(M_{p,(9 / 8)}\left(\|f\|_{E}\right)(x)+M_{p,(3 / 2)}\left(\|T f\|_{F}\right)(x)+T_{*} f(x)\right) .
\end{aligned}
$$

On the other hand, for all doubling cubes $Q \subset R$ with $x \in Q$ such that $K_{Q, R} \leq P_{0}$, where $P_{0}$ is the constant in Lemma 9.3 in [9], by (6) we have

$$
\left\|h_{Q}-h_{R}\right\|_{F} \leq c K_{Q, R}\|b\|_{*}\left(M_{p,(9 / 8)}\left(\|f\|_{E}\right)(x)+T_{*} f(x)\right) P_{0} .
$$

So by Lemma 9.3 in [9] we get

$$
\left\|h_{Q}-h_{R}\right\|_{F} \leq c K_{Q, R}\|b\|_{*}\left(M_{p,(9 / 8)}\left(\|f\|_{E}\right)(x)+T_{*} f(x)\right)
$$


for all doubling cubes $Q \subset R$ with $x \in Q$ and using (12) again, we obtain

$$
\begin{aligned}
& \left\|m_{Q}\left(C_{b} f\right)-m_{R}\left(C_{b} f\right)\right\|_{F} \\
& \quad \leq c K_{Q, R}\|b\|_{*}\left(M_{p,(9 / 8)}\left(\|f\|_{E}\right)(x)+M_{p,(3 / 2)}\left(\|T f\|_{F}\right)(x)+T_{*} f(x)\right) .
\end{aligned}
$$

From this estimate and (13), we get the sharp maximal function estimate.

Proof of the theorem 3.. Let $\left\{b_{Q}\right\}_{Q}$ be a family of numbers satisfying

$$
\int_{Q}\left|b-b_{Q}\right| d \mu \leq 2 \mu(2 Q)\|b\|_{* *}
$$

for any cube $Q$, and

$$
\left|b_{Q}-b_{R}\right| \leq 2 K_{Q, R}\|b\|_{* *}
$$

for all cubes $Q \subset R$. For any cube $Q$ and positive function $f$, we denote

$$
w_{Q}:=m_{Q}\left(V\left(\left|b-b_{Q}\right| f \chi_{\mathbb{R}^{d} \backslash \frac{4}{3} Q}\right)\right) .
$$

We can analogously prove that for all $p \in(1, \infty)$,

$$
\begin{aligned}
& \frac{1}{\mu\left(\frac{3}{2} Q\right)} \int_{Q}\left\|V_{b}^{+} f-w_{Q}\right\|_{F} d \mu \leq c\|b\|_{*}\left(M_{p,(9 / 8)} f(x)\right. \\
& \left.\quad+M_{p,(3 / 2)}\left(\|V f\|_{F}\right)(x)\right)
\end{aligned}
$$

for all $x$ and $Q$ with $x \in Q$, and

$$
\left\|w_{Q}-w_{R}\right\|_{F} \leq c\|b\|_{*}\left(M_{p,(9 / 8)} f(x)+\|V f(x)\|_{F}\right) K_{Q, R}^{2}
$$

for all cubes $Q \subset R$ with $x \in Q$. By (14) and (15), we obtain the following sharp maximal function estimate

$$
M_{F}^{\#}\left(V_{b}^{+} f\right)(x) \leq c_{p}\|b\|_{*}\left(M_{p,(9 / 8)} f(x)+M_{p,(3 / 2)}\left(\|V f\|_{F}\right)(x)+\|V f(x)\|_{F}\right) .
$$

Then, if we take $r$ such that $1<r<p<\infty$, we can get

$$
\begin{aligned}
& \left\|V_{b}^{+} f\right\|_{L_{F}^{p}(\mu)} \leq\left\|N_{F}\left(V_{b}^{+} f\right)\right\|_{L^{p}(\mu)} \leq c\left\|M_{F}^{\#}\left(V_{b}^{+} f\right)\right\|_{L^{p}(\mu)} \\
& \quad \leq c\|b\|_{*}\left(\left\|M_{r,(9 / 8)} f\right\|_{L^{p}(\mu)}+\left\|M_{r,(3 / 2)}\left(\|V f\|_{F}\right)\right\|_{L^{p}(\mu)}+\|V f\|_{L_{F}^{p}(\mu)}\right) \\
& \quad \leq c\|b\|_{*}\|f\|_{L^{p}(\mu)} .
\end{aligned}
$$




\section{REFERENCES}

1. J. García-Cuerva and J. Martell, Weighted inequalities and vector-valued CalderónZygmund operators on non-homogeneous spaces, Publ. Mat., 44 (2000), 613-640.

2. J. García-Cuerva and J. Martell, On the existence of principal values for Cauchy integral on weighted Lebesgue spaces for non-doubling measures, J. Fourier Anal. Appl., 7 (2001), 469-487.

3. J. Mateu, P. Mattila, A. Nicolau and J. Orobitg, BMO for nondoubling measures, Duke Math. J., 102 (2000), 533-565.

4. F. Nazarov, S. Treil and A. Volberg, Cauchy integral and Calderón-Zygmund operators on nonhomogeneous spaces, Internat. Math. Res. Notices, 15 (1997), 703-726.

5. F. Nazarov, S. Treil and A. Volberg, Weak type estimates and Calderon-Zygmund operators on nonhomogeneous spaces, Internat. Math. Res. Notices, 9 (1998), 463-487.

6. C. Segovia and J. L. Torrea, Weighted inequalities for commutators of fractional and singular integrals, Publ. Mat., 35 (1991), 209-235.

7. X. Tolsa, $L^{2}$-boundedness of the Cauchy integral operator for continuous measures, Duke Math. J., 98 (1999), 269-304.

8. X. Tolsa, Cotlar's inequality and existence of principal values for the Cauchy integral without the doubling condition, J. Reine Angew. Math., 502 (1998), 199-235.

9. X. Tolsa, BMO, $H^{1}$ and Calderón-Zygmund operators for non doubling measures, Math. Ann., 319 (2001), 89-149.

Wengu Chen and Changxing Miao

Institute of Applied Physics and Computational Mathematics,

P. O. Box 8009 ,

Beijing 100088,

P. R. China

E-mail: chenwg@iapcm.ac.cn

E-mail: miao_changxing@iapcm.ac.cn 